\title{
Primary angiosarcoma of the lung and pleura
}

\author{
G.C. Maglaras 1 , S. Katsenos 1 , J. Kakadelis², C. Katsanos ${ }^{1}$, Z. Metafratzi ${ }^{3}$, \\ D.G. Stefanou 4 , M.P. Vassiliou1 1 , S.H. Constantopoulos ${ }^{1}$
}

ABSTRACT: Primary angiosarcoma of the lung and pleura. G.C. Maglaras, S. Katsenos, J. Kakadelis, C. Katsanos, Z. Metafratzi, D.G. Stefanou, M.P. Vassiliou, S.H. Constantopoulos.

A 46 year old male smoker was admitted for severe continuing hemoptysis. Chest-X-rays and chest computed tomography revealed nodular infiltrates and bilateral hemothorax. Fiberoptic bronchoscopy resulted to non-diagnostic cytological and microbiological findings. Open lung and pleural biopsies after right thoracotomy revealed ep- ithelioid angiosarcoma and further staging assessment showed secondary brain and liver foci. The patient received several courses of chemotherapy but he died one month later.

The clinical, radiological, pathological, histochemical and therapeutic aspects of the disease are discussed.

Angiosarcoma, a rare tumour with poor prognosis should be taken into consideration in the differential diagnosis of hemoptysis.

Monaldi Arch Chest Dis 2004; 61: 4, 234-236.

Keywords: Hemoptysis, imaging findings, angiosarcoma.

${ }^{1}$ Department of Pneumonology, University Hospital of Ioannina.

2 Thoracic Surgery Unit, "G. Hatzikosta" General Hospital of Ioannina.

3 Department of Radiology, University Hospital of Ioannina.

4 Department of Pathology, University Hospital of Ioannina, Greece.

Correspondence: Stamatis Katsenos, MD; 3, Ierarhou Panaretou St; 45445,Ioannina, Greece; e-mail: skatsenos@yahoo.gr

\section{Case report}

A 46 year old male, heavy smoker $(90$ pack/year), working as a postman was admitted to the Pneumonology Department of the Ioannina University Hospital presenting continuing intermittent hemoptysis a month before his admission, which was not influenced by several courses of antibiotics.

Clinical examination of the chest revealed diminished breath sounds in the right middle and lower lung fields. Blood tests showed normochromic,

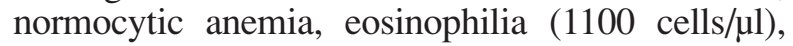
slightly increased erythrocyte sedimentation rate $(25 \mathrm{~mm} / \mathrm{h})$ and moderate hypoxemia $\left(\mathrm{PaO}_{2}: 64 \mathrm{~mm}\right.$ $\mathrm{Hg})$. Mantoux skin test was positive (15mm). Tests for serum tumour markers, rheumatoid factor, serum complement, antinuclear antibodies, antineutrophil cytoplasmatic antibodies and glomerular basement membrane antibodies were negative. Pulmonary function tests showed mild obstructive disorder (small airways disease) compatible with the patients' smoking habits, while lungs diffusing capacity (single breath $\mathrm{CO}$ test) was normal.

The chest-X-ray taken on admission showed a pleural based extensive alveolar consolidation in the right upper lobe (fig. 1a). Helical computed tomography (CT) performed 3 days after admission revealed a patchy consolidate in the right upper lobe and diffuse ground glass appearance especially in both lower lung fields (fig. 1b). Spiral CT pulmonary arterial angiography performed simultaneously was negative for emboli in pulmonary arterial trunk and the following branching system.

The patient underwent fiberoptic bronchoscopy (FOB). No intraluminal lesions were observed. Fresh blood of moderate quantity was coming from the right upper lobe bronchus. Bronchial washings were negative for mycobacteria and other common pathogens. Cytologic examination was also negative.

The patient continued to expectorate large quantities of blood (40 cc per day) and developed progressively increasing dyspnea on exertion. A new FOB was performed ( 7 days after the $1^{\text {st }}$ FOB) showing active bleeding from both upper lobe bronchi and the bronchus of the right lower lobe. A new chest-X-ray and high-resolution CT

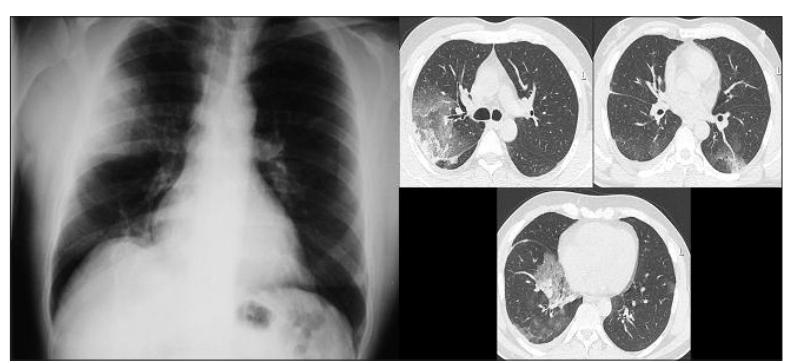

Fig. 1. - Chest-X-ray on admission: Pleural based extensive alveolar consolidation in the right upper lobe. Computed chest tomography (3 days after patient's admission): Patchy consolidate in the right uper lobe and diffuse ground glass appearance mostly in both lower lung fields. 
(fig. 2) performed on the $15^{\text {th }}$ day after the patient's admission showed further aggravation with bilateral pleural effusions, diffuse ground glass appearance and multiple focal consolidated patches in both lungs. Thoracentesis showed hemothorax. Cytology and biochemical study of the effusion and pathology of pleural biopsy specimens were not diagnostic.

The patient was transferred to the thoracic surgery department. Right thoracotomy was performed and specimens were obtained from both lungs and pleura. The histopathology refers to one pleural and three lung specimens showing large neoplastic infiltrations with medium and large sized cells. They have abundant eosinophilic cytoplasm with round nuclei and large mainly single

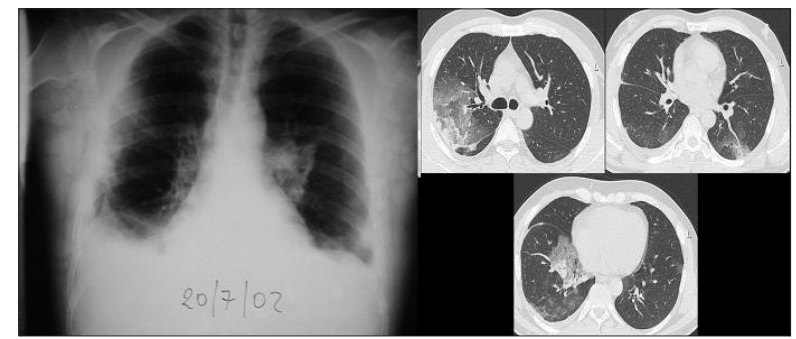

Fig. 2. - Chest-X-ray and computed tomography during patient's clinical deterioration: Bilateral pleural effusions, diffuse ground glass appearance and multiple focal consolidated patches in both lungs.

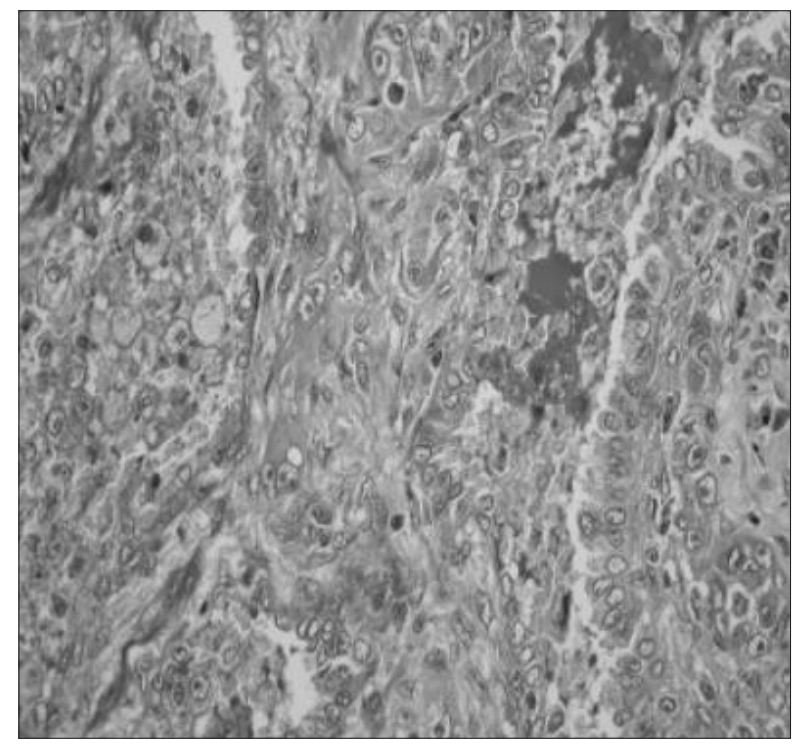

Fig. 3. - Lung and pleural biopsy: Immunohistochemical staining of the lung and pleural biopsy specimens with anti-CD31 monoclonal antibody and vimentin. The magnification scale bar depicts $200 \mu \mathrm{m}$.

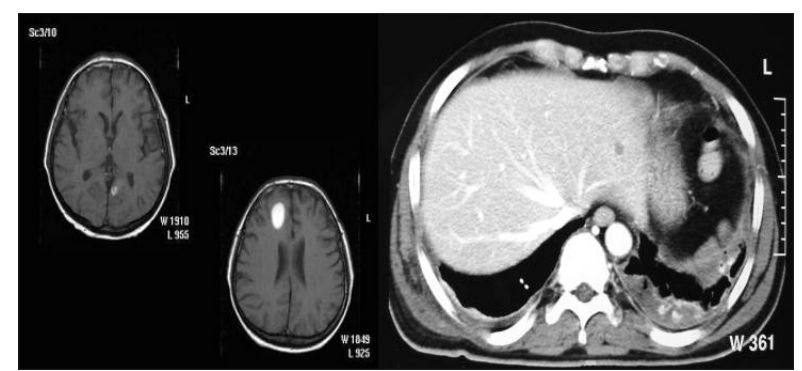

Fig. 4. - Brain, and upper abdomen computed tomography with characteristic brain and hepatic metastatic lesion. nucleoli. Eosinophilic intracellular round bodies and granules are recognized in some of the cells with or without isolated red blood cells, which form large compact bodies or obstruct the vessels lumen. The number of mitoses varies from 0 to 1 per optic high magnification field. The substratum is collagenoid with extravasculated red blood cells, and hemosiderin deposits inside and outside of macrophages. Foci of hemorrhagic or necrotic infiltration have also been recognized (fig. 3). According to the immunohistochemical study neoplastic cells were positive to vimentin and CD31 antigens and negative to pancytokeratin, keratin, epithelial membrane antigen (EMA) carcinoembryonic antigen (CEA), S-100 protein, Leu-M, VIII factor, CD34, common leucocytic, desmine and neuroid enolase (NSE). The findings correspond to epitheliod angiosarcoma, grade of malignancy II.

Abdominal and brain computed tomography were assessed for staging and revealed secondary brain and hepatic lesions (fig. 4).

A two-drug combination chemotherapy regimen was administered including courses of adriamycine and ifosfamide, with remission of his main symptom (hemoptysis) only for a short period of time. The patient's deterioration was rapid with continuing and progressively increasing hemoptysis and a fatal outcome within 3 months.

\section{Discussion}

Angiosarcoma is a rare malignancy corresponding to $2 \%$ of all soft tissue sarcomas, originating from vascular endothelium and mainly involving superficial soft tissue $(60 \%)$ and liver $(17 \%)[1,2,9]$. Primary intrathoracic angiosarcoma represents less than $1 \%$ of sarcomas and $0,02 \%$ of malignant primary lung tumours [9]. A slightly higher incidence is mentioned for males and middle aged [9]. Association of angiosarcoma with various agents is referred, such as exposure to thorotrast, vinyl chloride and insecticides, anabolic steroids or synthetic oestrogens intake and radiation treatment [9]. None of these agents could be associated with the present patient's malignancy.

Diagnosis of respiratory system epithelioid angiosarcoma necessitates exclusion of metastatic disease. Hemoptysis, dyspnea and chest pains are the more frequent manifestations. Radiological findings include multiple lung nodules, linear infiltrates, large solitary mass, pleural effusion, pleural thickness with nodular appearance, diffuse alveolar pattern and pneumothorax. Nevertheless, cases of pulmonary angiosarcoma with normal chest-X-ray have also been described. Diffuse ground glass appearance in both lungs is consistent with pulmonary hemorrhage and this was observed in our patient $[9,10]$. Biopsy and immunohistochemical study are necessary to establish the diagnosis. The disease is diagnosed by the presence of neoplastic cells stained strongly positive with the vascular endothelial markers CD31, CD34, factor VIII and vimentin. [8]

The clinical and laboratory course of the disease suggest that in our patient lung parenchyma 
was the primary tumour localization. Angiosarcoma of the lung is characterized by its dismal course and poor prognosis. Chemotherapy and radiotherapy are the usual therapeutic modalities. Surgical excision may be considered in case of solitary mass $[6,7]$. In general, response to chemotherapy is very poor, although recent studies suggest that liposomal doxorubicin seems more promising $[11,12]$.

In conclusion, primary angiosarcoma of the lung is a rare malignancy. Only 31 cases of pleural origin, 3 cases of pulmonary tissue origin, 2 cases of tracheal origin and 1 of pulmonary artery origin are reported $[3,4,5]$. Despite its low incidence, the disorder should be taken into account in the differential diagnosis of hemoptysis.

\section{References}

1. Junge K, Toens C, Peiper C, Hermanns B, Schumpelick V. Primary angiosarcoma of the lung. Chirurg 2001; 72 (8): 969-972

2. Girard C, Johnson WC, Graham JH. Cutaneous angiosarcoma. Cancer 1970; 26: 868-883.

3. Zhang PJ, Livolsi VA, Brooks JJ. Malignant epithelioid vascular tumors of the pleura : report of a series and literature review. Hum Pathol 2000; 31 (1): 29-34.

4. Tuppy H, Kolb R, Eckmayr J. Primary epithelioid an- giosarcoma of the proximal trachea. Pathologe 2000; 21: 460-463.

5. Goldblum JR, Rice TW. Epithelioid angiosarcoma of the pulmonary artery. Hum Pathol 1996; 26: 12751277.

6. Alexiou C, Clelland CA, Robinson D, Morgan WE. Primary angiosarcomas of the chest wall and pleura. Eur J Cardiothorac Surg 1998; 14 (5): 523-526.

7. Sheppard MN, Hansell DM, Du Bois RM. Primary epithelioid angiosarcoma of the lung presenting as pulmonary hemorrhage. Hum Pathol 1997; 28: 383-385.

8. Boucher LD, Swanson PE, Stanley MW, Silverman JF, Raab SS Geisinger KR. Cytology of angiosarcoma. Findings in fourteen fine-needle aspiration biopsy specimens and one pleural fluid specimen. Am J Clin Pathol 2000; 114 (2): 210-219.

9. Pui MH, Yu SP, Chen JD. Primary intrathoracic malignant fibrous histiocytoma and angiosarcoma. Australasian Radiology 1999; 43: 3-6.

10. Liu SF, Wu CC, Lai YF, Hsieh MJ. Massive hemoptysis and hemothorax caused by pleuropulmonary angiosarcoma. Am J Emerg Med 2002; 20 (4): 374-375.

11. Eiling S, Lischner S, Busch JO, Rothaupt D, Christophers E, Hauschild A. Complete remission of a radioresistant cutaneous angiosarcoma of the scalp by systemic treatment with liposomal doxorubicin. Br J Dermatol 2002; 147 (1): 150-153.

12. Budd GT. Management of angiosarcoma. Curr Oncol Rep 2002; 4 (6): 515-519. 